\title{
Violencias estructurales
} $y$ emergentes: suicidio
y Camelódromo
en Londrina, Brasil

\author{
Angela Golono de Deus* \\ Carla Maria Freres Stipp Baptista*** \\ Carolina Nunes França $a^{* * * *}$
}

\begin{abstract}
Como resultado de la investigación de campo realizada en el centro de la ciudad de Londrina, en el estado de Paraná, Brasil, se discuten las violencias estructurales y emergentes. En un primer momento, fue identificado un caso de violencia emergente generado por el modelo de desarrollo, asociado al suicidio. En un segundo momento, fueron identificados casos de violencia estructural en el Camelódromo y la presencia de camelós en el área.
\end{abstract}

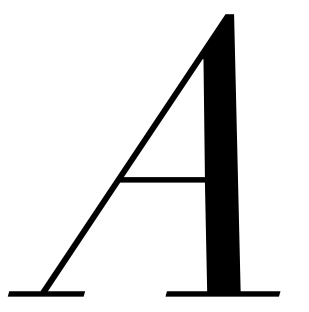

L PENSAR EN VIOLENCIA automáticamente se hace referencia a situaciones asociadas a la criminalidad que afectan la integridad física o bienes materiales de los individuos. Estudios recientes asociados al concepto de la seguridad humana han identificado diferentes formas de violencias clasificadas, principalmente, en

* Estudiante de la Maestría en Geografía de la Universidade Estadual de Londrina, Brasil. Correo: angelagolono@gmail.com.

** Estudiante de la Maestría en Geografía de la Universidade Estadual de Londrina, Brasil. Correo: carlastipp@hotmail.com.

**** Estudiante de la Maestría en Geografía de la Universidade Estadual de Londrina, Brasil. Correo ca.franca@hotmail.com.

Traducción del portugués de Guadalupe Margarita González Hernández. dos grupos: violencias emergentes, que son aquellas relacionadas con la criminalidad, fácilmente identificadas por su manifestación física o psicológica de forma superficial; y las violencias estructurales, fomentadas por mecanismos afiliados al modelo de desarrollo y que no son percibidas por las personas como un tipo de violencia.

Bajo esas prerrogativas es que en el presente artículo se realizará a priori una discusión teórica acerca del concepto de violencia y cómo se expresa en el medio urbano, pudiendo ser causada por factores estructurales asociados a la estructura organizativa de las ciudades, o por factores externos estrictamente relacionados con el modelo de desarrollo económico vigente.

Con la finalidad de identificar y reflexionar sobre situaciones y casos de violencias emergentes y estructurales en la ciudad de Londrina, se realizó trabajo de 
campo en el Centro Histórico, donde se sitúan una cantidad importante de comercios y servicios. Durante dicha investigación se aprecia el hecho de que la violencia puede estar oculta y arraigada en la organización de la ciudad, siendo necesario analizar los factores que están atrás de su configuración territorial.

El municipio de Londrina está localizado al norte del estado de Paraná, al sur de Brasil, y su perímetro urbano - la ciudad de Londrina- se finca, igualmente, al norte del municipio (véase figura i) y albergó, según datos del 2012, aproximadamente a 493,520 residentes, de los 5o6, zor habitantes municipales.

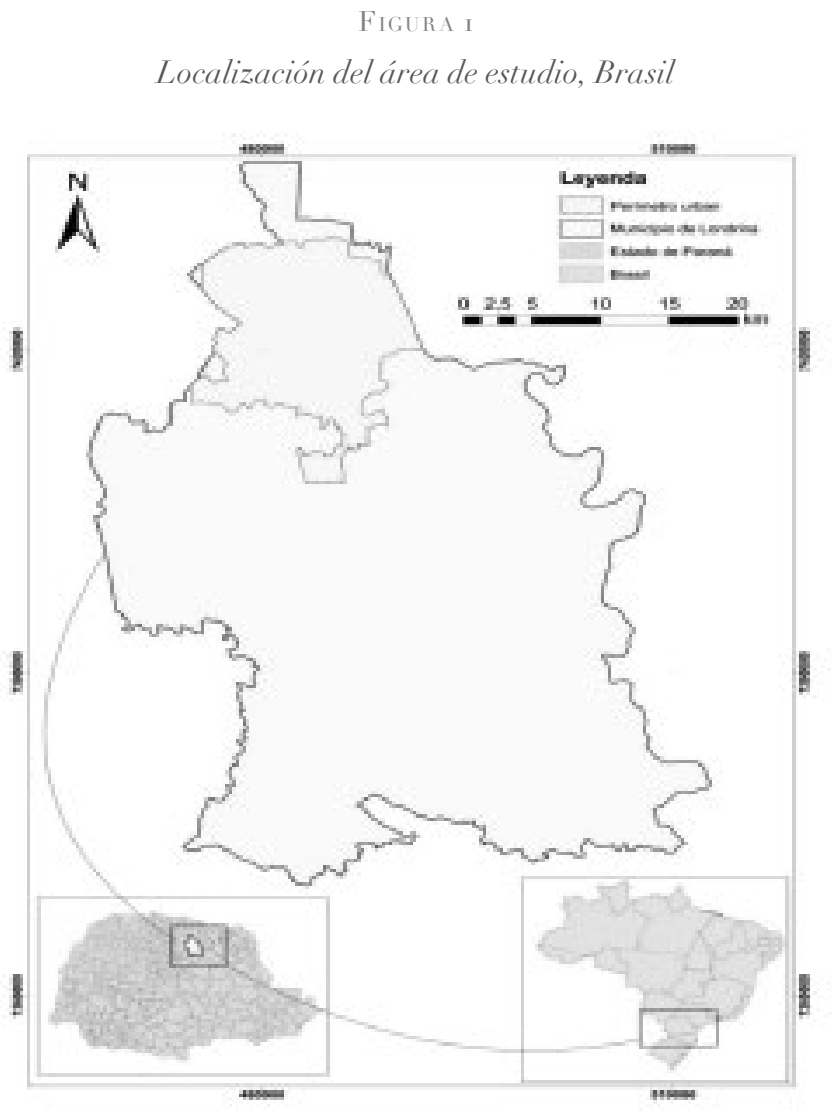

Fuente: Carolina Nunes França, 20ı^.

Creada en 1929 como parte del emprendimiento de la Compañía de Tierras del Norte de Paraná (Companhia de Terras Norte do Paraná, CTNP), la ciudad de Londrina fue concebida teóricamente a través de un plan donde sólo cubriría las necesidades básicas de los propietarios de la tierra y la población rural. Desde sus inicios, Londrina poseyó dinámica mercantil al liderar la producción mundial del café y sus consecuentes negocios con la tierra; situación que provocó que el plan original fuera rápidamente superado bajo el crecimiento físico y territorial de la zona urbana. ${ }^{\prime}$
El origen de la ciudad, proyectada para 20 mil habitantes, tuvo sus límites extrapolados por el surgimiento de nuevos asentamientos, aumento del comercio y creación de nuevas vías de tránsito, en especial, la construcción de las del ferrocarril en I934. ${ }^{2}$

En la década de los años ochenta, las regiones norte y sur de la ciudad comienzan a ser ocupadas por la construcción de conjuntos habitacionales verticales. Dichos conjuntos fueron distribuidos de forma desigual y distantes al centro de la ciudad, intercalados con grandes espacios baldíos. El mejoramiento de la infraestructura y equipamiento urbano, así como la atracción de industrias de tecnología de punta y las diferencias sociales y ambientales, fueron fruto de ese proceso de expansión urbana y económica. ${ }^{3}$ Actualmente la ciudad se considera un polo regional, económico, cultural y de servicios.

Todo ese proceso urbanístico, en un corto periodo, provocó segregación social, fragmentación espacial y diferentes tipos de violencia urbana que pueden ser comprendidas en el presente.

\section{Violencias EMERGENTES}

Las violencias emergentes son aquellas asociadas a la criminalidad, tienen concepción y origen punitivo, y son provocadas ya sea por el modelo de desarrollo o por la organización autónoma de la vida urbana. Estas violencias se manifiestan principalmente como fenómenos urbanos, aunque no necesariamente lo sean.

Son violencias emergentes producto del modelo de desarrollo las que se expresan de forma directa, visible y sentida, que involucran a un agresor y una víctima claramente identificables ${ }^{4}$ como, por ejemplo, robo a mano armada, de bienes muebles o inmuebles, disputas por tráfico de drogas, suicidio, entre otras. La mayoría de estas violencias se manifiestan espacialmente en las zonas urbanas, pero no son producto de la organización de dicha ciudad, sino del modelo de desarrollo, por tanto son violencias emergentes urbanizadas.

En tanto las violencias emergentes generadas por la ciudad, llamadas violencias emergentes urbanas, refieren a las violencias que impone espacialmente un grupo social urbano a otros como sinónimo de exclusión social, como por ejemplo la xenofobia. En el Código Penal Brasileño, este tipo de violencias, ya sean meramente urbanas o producto del modelo de desarrollo, son tipificadas como criminales. 
VIOLENCIA EMERGENTE URBANIZADA: SUICIDIO

Durante el trabajo de campo realizado en el centro de la ciudad de Londrina a mediados de agosto de 20I/4, se percibieron dos tipos de violencia emergente. El primero refiere al suicidio, considerado como la eliminación directa o destrucción de la vida propia. Por imposibilidad de penalizar el suicidio, no se incrimina su práctica para el cumplimiento de un bien disponible, o sea la vida; el acto ilegal se centra en que la ley beneficia la coacción para impedirlo. Por tanto, incrimina sólo hechos y situaciones donde cualquier persona ayude o influya en el suicidio de otro. ${ }^{5}$

Los factores que llevan a este tipo de violencia emergente son generalmente provocados por el modelo de desarrollo socioeconómico excluyente y las formas como lo asimilan sus residentes. Este fenómeno es característico de las ciudades de tamaño medio o grande:

El suicidio es entendido como producto o reflexión de las alteraciones sociales y económicas ocurridas en las sociedades. En realidad, ese mecanismo de causa no se da de forma directa, sino como resultado de una serie de factores. Esto es particularmente visible en regiones con rápida transformación, o que comprometen y alteran no solamente la situación financiera de los sujetos, sino su vida en aspectos socioculturales. ${ }^{6}$

La ciudad de Londrina ha registrado varios casos de suicidio, en especial en el centro, donde se observa que el edificio Júlio Fuganti es el idóneo para realizarlo (véase figura 2). Este edificio en forma de triángulo, producto de la bifurcación de dos avenidas concentra actividades comerciales y de oficinas y es utilizado frecuentemente por aquellos que tienen el referido acto ilícito como objetivo. Desafortunadamente no hay mucha transparencia sobre su incidencia en las instituciones estatales involucradas, pero periódicos locales enuncian de tres a cuatro al año.

\section{Violencias estructurales} URBANIZADAS Y URBANAS

Asociadas al modelo de desarrollo o a la dinámica interna de las ciudades, las violencias estructurales no son claramente perceptibles por la población en cuanto a manifestación violenta. Según Blasco y Parra, ${ }^{7}$ la violencia estructural puede ser denominada también como violencia sistémica, oculta, indirecta e institucional, y es resultado del proceso de desigualdad, causado por injusticias sociales o perjuicios al bienestar humano en términos de necesidades básicas.

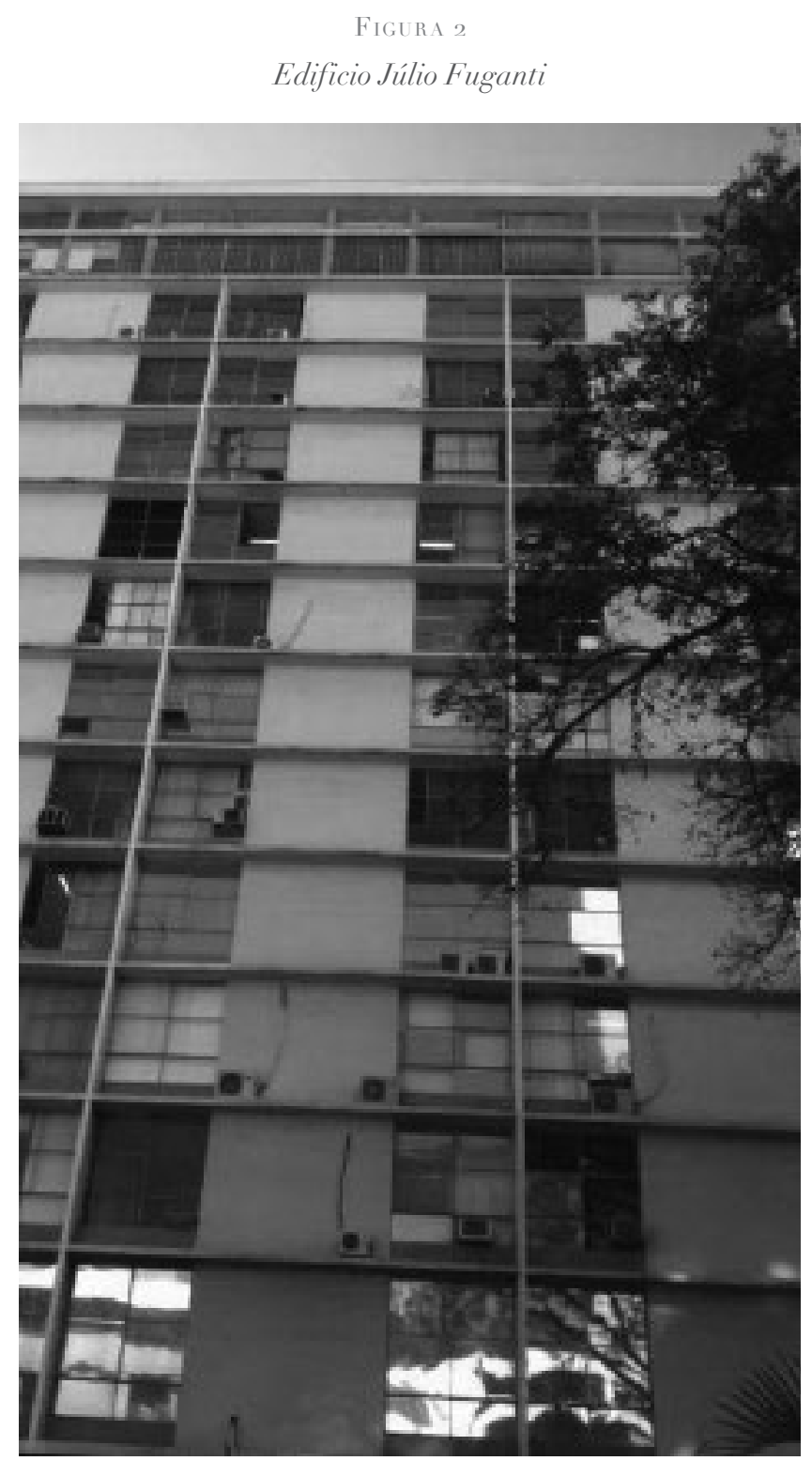

Fotografía: Carla Maria Freres Stipp Baptista, 20I/.

A la violencia estructural o sistémica que es causada por el modelo de desarrollo y manifestada espacialmente en la ciudad, se le llama violencia sistémica urbanizada. Se expresa como los cambios surgidos en la economía y sociedad que provocan mudanzas en el espacio urbano; por ejemplo, la reestructuración espacial que sufren las ciudades cuando ha ocurrido una desindustrialización o un cambio del papel del Estado en la dinámica socioeconómica que pronuncia la ur- 
banización de la pobreza o accesibilidad desigual a centros de consumo o trabajo, etcétera.

Producto de la organización interna de las ciudades, las violencias estructurales urbanas se revelan en la forma en que se construye la fisonomía urbana, se sitúa espacialmente la estructura social dando lugar a la segregación socioespacial, fragmentación social, acceso diferenciado de los recursos urbanos o privatización de servicios públicos.

En el centro de Londrina se visualizaron algunos ejemplos de estas violencias en la localización espacial del trabajo informal -comercios ambulantes o en los Camelódromos-.Ambos son resultado del desempleo y formas renovadas de producción de pobreza urbana. A pesar de la recuperación del empleo y mejoramiento de las condiciones económicas nacionales en los últimos años, se ha incrementado el número de personas sometidas al trabajo informal.

\section{Violencia ESTRUCTURAL URBANIZADA:} VENDEDORES AMBULANTES

Los comerciantes ambulantes se instalan en las principales avenidas del centro y venden objetos baratos $y$, muchas veces, ilegales (véanse figuras 3 y 4). Es una violencia estructural urbanizada causada por el modelo de desarrollo, una vez que los orilla a trabajar de manera informal sin la estructura legal y laboral necesaria.

Con el objetivo de categorizar el marco de los vendedores ambulantes en Brasil, la ley complementaria 128/2008 ampara al microemprendedor individual y formaliza su trabajo, reconociendo que las causas de su manifestación están en el desempleo y las exigencias del mercado de trabajo. ${ }^{8}$ Por tanto, las autoridades gubernamentales deben implementar acciones o proyectos que permitan disminuir este fenómeno.

Según Déniz y colaboradores, ${ }^{9}$ de 3 o vendedores ambulantes entrevistados en 2009, apenas I7 por ciento son formales, siendo sólo io por ciento de acuerdo con dicha ley. El 65 por ciento nunca ha oído algo sobre esa ley y 27 por ciento no pretende salir de su estado de informalidad. El principal motivo que lleva a los ambulantes a no cambiar su estatus es el rechazo al pago de impuestos (42 por ciento de los entrevistados) y la satisfacción y comodidad de su situación actual $(37$ por ciento), siendo que éste segundo grupo está formado por quienes trabajan por la necesidad de una fuente de ingresos, forzados por el desempleo.
Figura 3

Ambulantes en el centro de Londrina

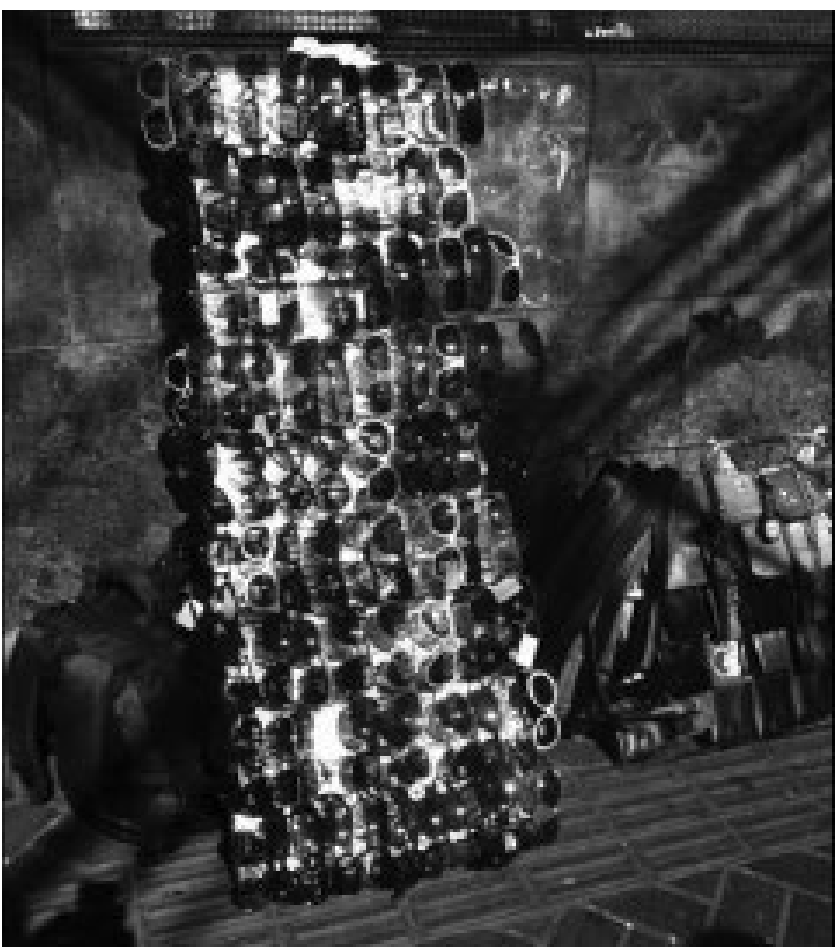

Fotografía: Carla Maria Freres Stipp Baptista, 2014.

FigURA 4

Ambulantes en el centro de Londrina

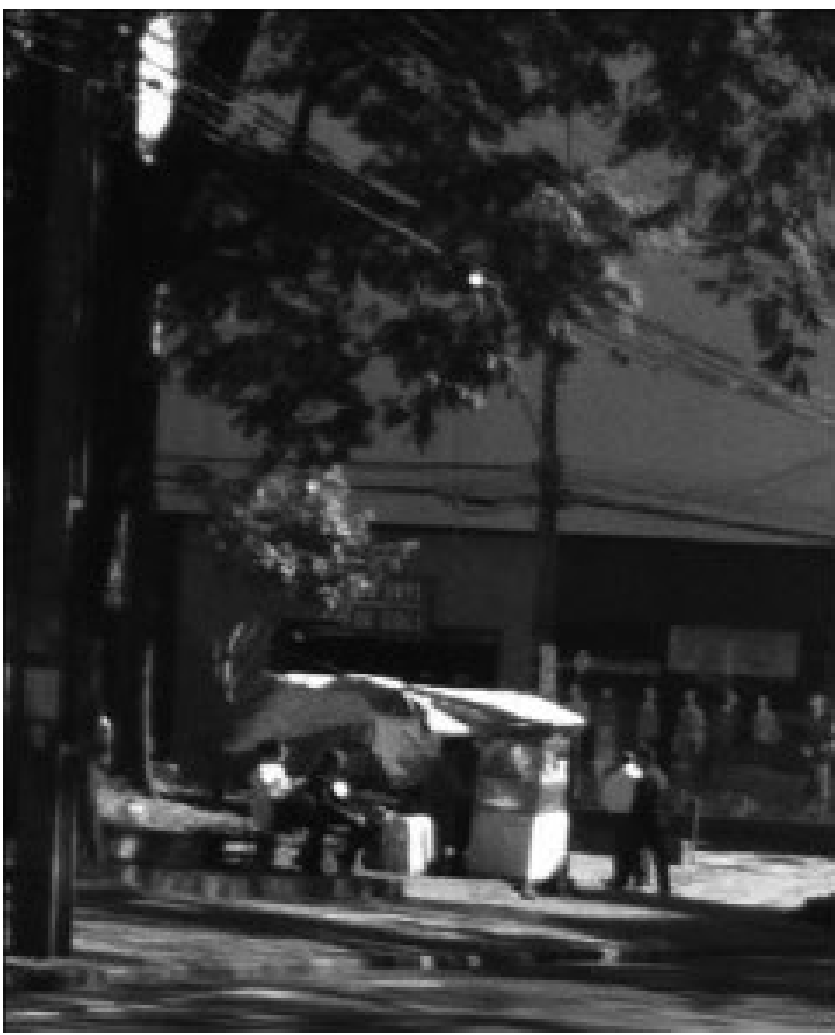

Fotografía: Carla Maria Freres Stipp Baptista, 20I4. 
Camelódromos

En Londrina, los primeros vendedores ambulantes comenzaron con la comercialización de productos diversos a mediados de la década de los noventa. Como se observa en la figura 5 , las primeras instalaciones, situadas próximas a la terminal de autobuses de la ciudad, eran improvisadas y precarias, se resumían en una larga fila de go tiendas improvisadas, con dimensiones inferiores a i.5 metros de largo. ${ }^{\circ}$

Durante la década de los noventa, el incremento de personas desempleadas propició el mismo comportamiento en servicios y comercios informales. Adicionalmente, las transformaciones urbano-fronterizas sufridas en Foz de Iguaçu y Ciudad del Este por el flujo turístico y comercial internacional a gran escala estimularon el incremento comercial de productos fronterizos en Londrina;" situaciones que dispararon los reclamos de comerciantes establecidos que se sentían afectados por la competencia y de personas descontentas por la localización de los ambulantes en las importantes avenidas de la ciudad, generando la preocupación del gobierno municipal por relocalizarlos y mejorar la estructura de las tiendas.
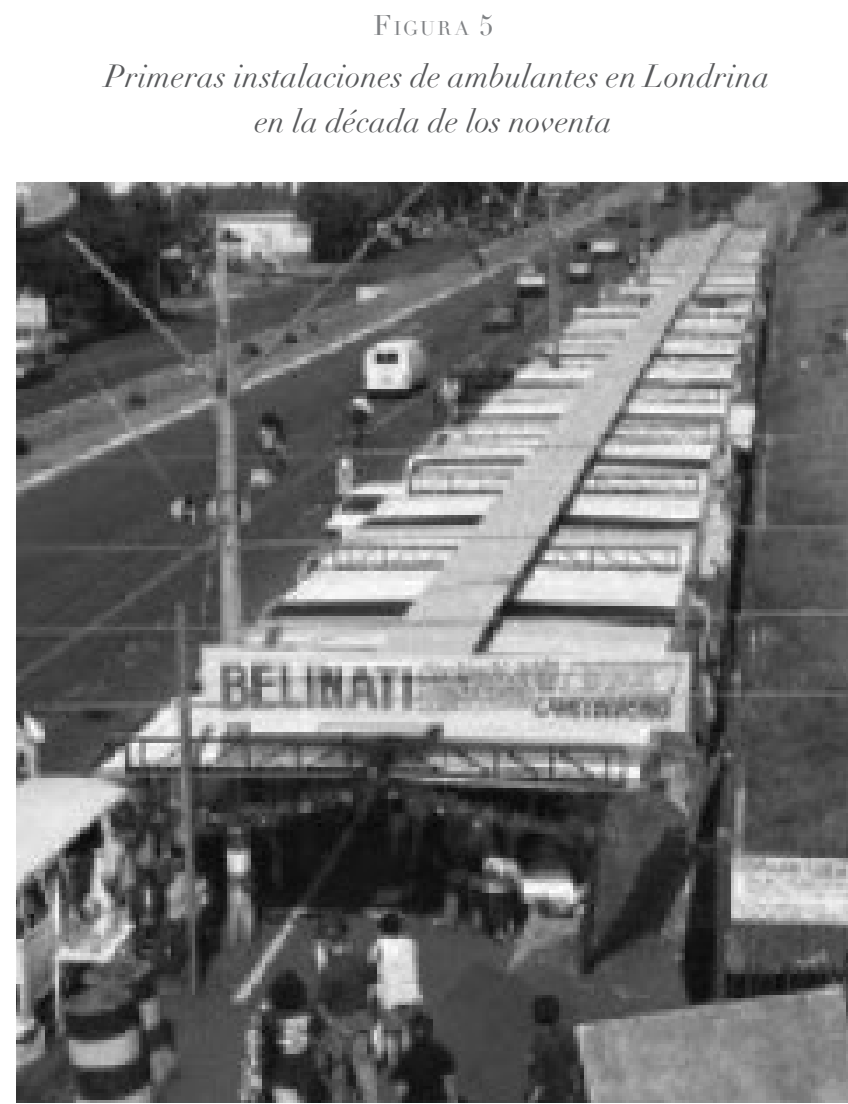

Fuente: Oliveira, 2oog.
A inicios del siglo XXI, las discusiones por su localización fueron tomando más fuerza y las tensiones entre ambulantes y el poder público se intensificaron. El resultado fue el proyecto de construcción de un Camelódromo formalizado, inaugurado en enero de 2003 en el centro de la ciudad de Londrina. Los comerciantes pasaron por un riguroso registro, evitando la permanencia de los comerciantes que vendían productos de terceros. ${ }^{2}$

Figura 6 Interior del Camelódromo de Londrina

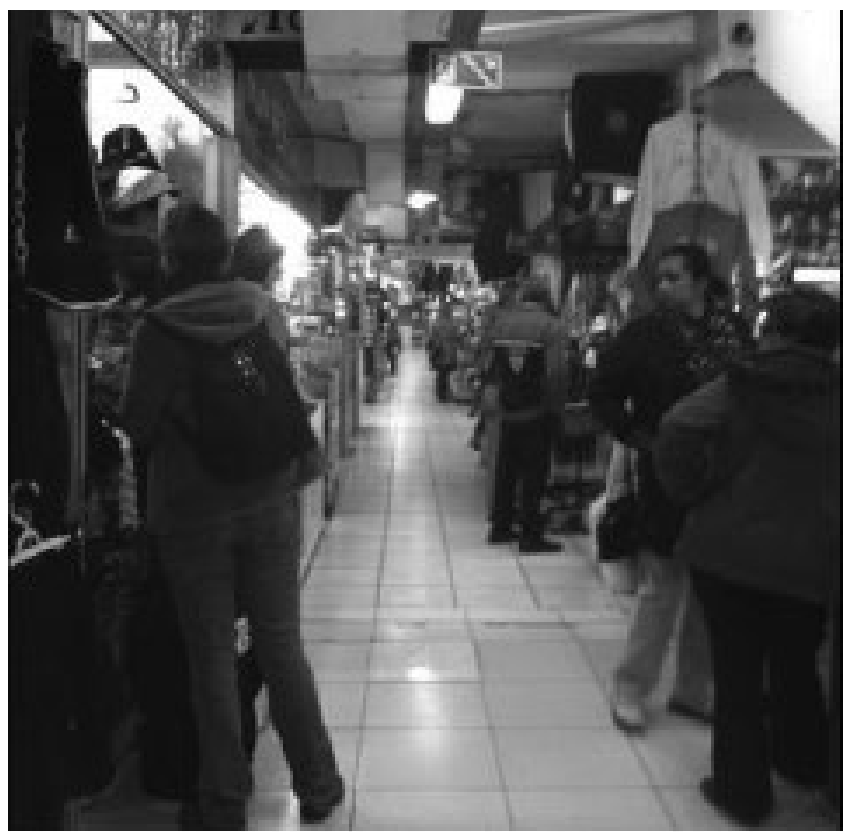

Fotografía: Carla Maria Freres Stipp Baptista, 2014.

Después de una década de funcionamiento, el Camelódromo cuenta con más de 3 oo pequeños establecimientos comerciales, donde predomina la comercialización al menudeo de ropa y electrónicos (véase figura 6). Los propietarios de los establecimientos están directamente relacionados con las actividades comerciales diarias de los londrinenses y se encargan de la atención o contratación de personal de venta y limpieza del local. ${ }^{3}$ Se estima que el Camelódromo es responsable de la generación de más de 800 empleos directos e indirectos. ${ }^{14}$

Este lugar aún es visto de manera despreciable por una parte de la población londrinense y en especial por los comerciantes establecidos. Son etiquetados como la extensión del crimen organizado, por comercializar productos ilegales oriundos de las ciudades fronterizas de Paraguay con Brasil, así como, en menor escala, importados de China. El discurso del impacto negativo del comercio ambulante se sobrepone entre 
los londrinenses a los problemas estructurales del modelo de desarrollo que acarrea y sobrevive la desigualdad social y los problemas sociales asociados..$^{\check{2}}$

Con una perspectiva histórica, el Camelódromo de Londrina, una vez construido en 2002, fue concebido como una solución para un creciente número de comerciantes ambulantes que trabajaban en la zona central, objeto de reclamaciones de los demás comerciantes de la ciudad y visto con prejuicio por muchas personas que consideran este sitio como "un lugar donde compran los pobres". Adicionalmente, su localización no afecta los intereses inmobiliarios y de imagen urbana del centro de Londrina: a pesar de su localización central, se encuentra en las calles más transitadas por personas de bajos ingresos y transporte público; lejos de recién renovados y regenerados espacios para comercios y servicios establecidos apreciados y solicitados por consumidores londrinenses de alto y medio ingreso. El Camelódromo es una forma de exclusión, resultado de las consecuencias de la desigualdad social -producto del sistema- y reforzado por la propia planeación urbana que produce y reproduce formas de segregación espacial y fragmentación social.

\section{Consideraciones finales}

El concepto de seguridad humana, en el sentido amplio, debe incluir los aspectos de calidad de vida en donde es fundamental garantizar las condiciones básicas de vida. Bajo esta premisa, el presente trabajo evidenció la presencia de distintos tipos de violencia en la ciudad de Londrina, que están concretizados en su propia estructura económica y organización urbana, y por tanto, son violencias implícitas y sistémicas.

En la ciudad de Londrina, aunque las violencias emergentes urbanizadas sean recurrentes en los noticieros, fue posible observar que hay ocultamiento de información en la ocurrencia de casos de suicidio, siendo necesario buscar información en estudios que elaboran testimonios. Cuando se observan a fondo los motivos que están detrás de los casos de suicidio, es posible observar estrecha relación con la escasez de algunos aspectos que conjuran la calidad de vida. El suicidio se enfrentó con motivaciones que genera el sistema capitalista y donde las ciudades son la máxima expresión espacial de marginación, desigualdad social, prejuicios, falta de oportunidades y segregación.

Por medio del análisis confinado al entendimiento del sentido amplio de la seguridad humana, fue posible observar las violencias adyacentes al sistema y efectuadas por la planeación urbana, como es el caso de la segregación espacial y social que sufren los vendedores ambulantes y su localización comercial, efectuado esto por el poder público y aprobado por la mayoría de la sociedad londrinense.

\section{- notas $\cdot-$}

' Tânia Maria Fresca, (2002), "Mudanças recentes na expansão físico-territorial de Londrina”, Geografia (Londrina), vol. II, núm. 2, pp. 241-264.

'Andrei Olak Alves, (2005), Expansão Urbana de Londrina e Tendências à Metropolização, Londrina, Universidade Estadual de Londrina.

${ }^{3}$ Idem.

${ }^{4}$ José Blasco y Daniel Parra (2003), "Violencia estructural: una ilustración del concepto”, Documentación Social, vol. 5, núm. І3г, pp. $57-72$.

${ }_{5}^{5}$ Júlio Fabbrini Mirabete (200I), Manual de Direito Penal, São Paulo, Atlas.

${ }^{6}$ Keila Rodrigues Souza, Yonissa Marmitt Wadi y Jeferson Andronio Ramundo Staduto (2007), "Suicídio e Desenvolvimento Regional: Um estudo sócio-econômico da incidência da auto violencia nas mesorregiões oeste e sudeste do Paraná (IIgo a 2005)", XLV Congresso da Sociedade Brasileira de Economia, Administração e Sociologia Rural, Universidade Estadual de Londrina, p. 3.
7 José Blasco y Daniel Parra (2003), op. cit.

${ }^{8}$ Junior Deniz, Ivan Manhas Correia, Ricardor Xavier Galdino y Rodrigo Seiji Yamaguchi (20ro), "O reflexo da M.E.I. para os vendedores ambulantes de produtos alimentícios da região central da cidade de Londrina", Revista de Estudo Contábeis, vol. I, núm. I, pp. 77-89.

${ }_{9}$ Op. cit.

${ }^{10}$ Edilson Luis Oliveira (2009), Divisão do Trabalho e circuitos da economia urbana em Londrina -PR, São Paulo, Universidade de São Paulo.

"Alfredo Fonseca y Antonio Carolos Lignani (2003), "Um estudo sobre o eixo Cascavel-Foz do Iguaçu, na região oeste do Paraná", Revista Paranaense de Desenvolvimento, núm. ı́4, pp. 79-102.

12. Edilson Luis Oliveira (2009), op. cit.

${ }^{3}$ Idem.

14 Prefeitura de Londrina (2014) disponible en http://londrina.pr.gov.br consultado el 3i de agosto de 20I4.

เ5 Edilson Luis Oliveira, (2009), op. cit. 